\title{
On molecular and continuum boundary conditions for a miscible binary fluid
}

\author{
Colin Denniston and Mark O. Robbins \\ Department of Physics and Astronomy, The Johns Hopkins University, Baltimore, Maryland 21218, USA
}

(November 3, 2018)

\begin{abstract}
We show that molecular dynamics simulations can furnish useful boundary conditions at a solid surface bounding a two-component fluid. In contrast to some previous reports, convective-diffusive flow is consistent with continuum equations down to atomic scales. However, concentration gradients can produce flow without viscous dissipation that is inconsistent with the commonly used Navier slip condition. Also, differential wetting of the two components coupled to a concentration gradient can drive convective flows that could be used to make nano-pumps or motors.
\end{abstract}

83.50.Rp, 83.10.Rs, 47.60.+i,68.08.Bc

Hydrodynamic theories of flow past a solid surface need to assume boundary conditions for the fluid velocities at the surface. Such interfacial behavior is often very difficult to access experimentally. Recent simulation studies of fluids have revealed a range of boundary conditions for single component fluids and related them to the microscopic interactions [1,2]. However, there are many problems where the appropriate boundary conditions are still in doubt. These include flow near a moving contact line, the liquid crystal order parameter in the presence of flow, and convective-diffusive flow of miscible fluids.

In a recent letter [3] Koplik and Banavar studied two fluids that individually obeyed a "no-slip" boundary condition at the surface. In a mixture of these fluids, they found that the velocities of each component still vanished at the wall. They concluded that the boundary condition accompanying the convective-diffusive transport of a binary fluid mixture demands that the velocities of the two species be equal at the wall. It was subsequently pointed out [4] that this boundary condition contradicts Fick's law of molecular diffusion, at least in its usual form where the diffusion coefficient is assumed to be position independent. Two possibilities were suggested. The first [4], that the convective flows studied in Ref. [3] were large enough to mask the effect of diffusion. The second, that the continuum equations need to be modified near the surface in order to obtain consistent results [4,5].

In an attempt to resolve this issue, Brenner and Ganesan [6] undertook a singular perturbation analysis. A continuum inner region with a refined form of Fick's law on which the no-slip condition applies for both components was asymptotically matched to an outer region which follows the standard form of Fick's law. As the details of the refined form of Fick's law are still somewhat ambiguous, Brenner and Ganesan conclude that "simulations purporting to derive" boundary conditions "by direct probing of those molecules near to the wall will necessarily give rise to erroneous macroscale conclusions". Essentially, they argue that the simulations are useless since they do not give the information necessary to match up to the known equations for bulk fluids. If true, this would be a very disappointing situation.

In this paper we examine flow boundary conditions for binary fluid mixtures using molecular dynamics simulations in the regime where diffusive flow is either dominant or on the same scale as the convective flow. We find bulk flow consistent with Fick's law within one molecular diameter from the wall, and appropriate boundary conditions for the known equations of bulk fluids are readily obtained. If concentration gradients are present, the individual velocities of the two species are not equal at the wall. More surprisingly, we find that density gradients can lead to a net flow without viscous dissipation! We also find that the coupling of wetting potentials with concentration gradients can give rise to Marangoni-like convective flow that could be used to make nano-pumps or nano-motors.

We consider a mixture of two types of molecules, labeled $a$ and $b$, in a slit geometry similar to that used for a single fluid in Ref. [1]. There are two walls, at $z=0$ and $z=L$, and periodic boundary conditions in the $x-y$ plane. The walls contain type $w$ atoms fixed to lattice sites forming two (111) layers of an fcc surface. The interactions between atoms of type $i$ and $j$ separated by a distance $r$ are modeled using a Lennard-Jones (LJ) potential, $V_{i j}(r)=4 \epsilon_{i j}\left[\left(\sigma_{i j} / r\right)^{12}-\left(\sigma_{i j} / r\right)^{6}\right]$, where $\epsilon_{i j}$ specifies the interaction energy and $\sigma_{i j}$ the interaction length. Their averages are denoted by $\epsilon$ and $\sigma$, respectively. A characteristic time scale is given by $\tau=\sigma(m / \epsilon)^{1 / 2}$, where $m$ is the average of the molecular masses $m_{i}$. Unless specified, the force is truncated at $r_{c}=2.2 \sigma$ to improve computational efficiency.

To obtain a steady-state concentration gradient we artificially construct a direction dependent osmotic membrane at $x=0$. The membrane is less than one $\sigma$ thick, and preferentially transmits atoms of type $a$ from left to right and conversely for atoms of type $b$. In a typical simulation we wait $5000 \tau$ for the steady-state concentration gradient to establish itself, and then average over a subsequent 15000 to $150000 \tau$ to collect data. Note that these time scales are more than an order of magnitude greater than in Ref. [3], thus allowing much greater sen- 
sitivity. Most channels studied had $L=16.4 \sigma$, but we have also found similar results for systems twice as wide.

The diffusive flux $\mathbf{J}_{j}$ of particles of type $j$,

$$
\mathbf{J}_{j} \equiv \rho_{j}\left(\mathbf{v}_{j}-\mathbf{v}\right),
$$

is defined [7] relative to the barycentric, or mass averaged, velocity $\mathbf{v} \equiv\left(\sum_{i} \rho_{i} \mathbf{v}_{i}\right) / \rho$, where $\rho_{i}$ is the positiondependent mass density of species $i$, and $\rho \equiv \sum_{j} \rho_{j}$ is the total mass density. Fick's law relates the diffusive flux to the gradient of the concentration $c_{j} \equiv \rho_{j} / \rho$ [7],

$$
\mathbf{J}_{j}=-\rho D \nabla c_{j},
$$

where $D$ is the diffusion constant. The results of Refs. [3] 6] suggest that there may be a macroscopic layer where the diffusion constant depends on proximity to the wall.

For simplicity, we first consider a system where $\sigma_{i j}=\sigma$ for all interactions and $m_{a}=m_{b}=m$. All fluid-fluid $\epsilon_{i j}=\epsilon$, and the wall-fluid coupling $\epsilon_{w f} \equiv \epsilon_{w a}=\epsilon_{w b}$ is varied. In this case entropy is the only driving force. Figure 11(a) shows the resulting steady state densities $\rho_{j}$ of the two species. Both vary linearly and the total density is constant throughout the system. Since $a$ and $b$ particles have equal and opposite concentration gradients we find that their fluxes are equal and opposite and $\mathbf{v}=0$. If the difference between individual velocities vanished at the wall, as in Ref. 4, both fluxes would have to vanish there. Figure 1(b) illustrates that the fluxes may actually increase at the wall. To eliminate variations with $x$ we plot $D=v_{x, j}\left(c_{j} / \partial_{x} c_{j}\right)$ as a function of height $z$ for three different wall-fluid interaction strengths. The diffusion constant, and thus the flux, goes down near the wall for $\epsilon_{w f} / \epsilon=1.0$, remains constant for $\epsilon_{w f} / \epsilon=0.3$, and goes up for $\epsilon_{w f} / \epsilon=0.1$. Thus, for $\epsilon_{w f} / \epsilon=0.1$, the difference between the individual velocities of the two species is actually highest at the wall. The value of $D$ is normalized by the bulk diffusion constant which was calculated for a system where the walls were replaced by periodic boundary conditions in the $z$ direction. As can be seen, there is no statistical difference from bulk diffusion at distances more than about one molecular diameter from the wall. Thus, in contrast to References [3] 6], there is no need to supplement Fick's law with a continuum boundary layer which obeys a modified constitutive relation.

Earlier work [1,2] on single component LJ fluids is well described by the Navier boundary condition, which assumes that the velocity difference between solid and fluid is proportional to the viscous shear stress. For our geometry this implies

$$
\left.v_{x}\right|_{w a l l}=\left.L_{s} \partial_{z} v_{x}\right|_{w a l l}
$$

where $L_{s}$ is called the slip length. A larger value of $L_{s}$ implies less drag at the interface, and we find that it correlates with greater diffusion at the wall. In most cases, the slip length has atomic dimensions [1, 2], and it is less than $2 \sigma$ for single fluids with the walls considered here.
The Navier condition presupposes that in the purely diffusive case, where $\partial_{z} v_{x}=0$, the barycentric velocity vanishes identically. However, Fig. 2(a) shows that this is not necessarily the case. For this simulation the masses were changed to $m_{a}=0.75 m$ and $m_{b}=1.25 m$ and $\epsilon_{w f} / \epsilon=1.0$. We find that the number densities $N_{i}$ are the same, within statistical errors, as those of Fig. 1 (a) and the mean velocities of each species are also unchanged. However, due to the mass difference, there is now a mass density gradient and a small net mass flux $\mathbf{J}=\rho \mathbf{v}$ along the channel (Fig. 2(a)). One might wonder if the Navier condition still applies, but with a very small velocity gradient and very large slip length. However, both single and two-component simulations of purely convective flow for the same parameters yield "stick" boundary conditions, i.e. the effective "wall" position is inside the fluid and $L_{s}$ is essentially zero. Moreover, there is no measurable viscous stress and the entire flux profile follows from a general continuum relation that we now describe.

For the example of Fig. 2(a) the mean molar velocity $\mathbf{v}^{m} \equiv\left(\sum_{i} N_{i} \mathbf{v}_{i}\right) / N$ vanishes, where $N_{i}$ is the molar density of component $i$ and $N \equiv \sum_{i} N_{i}$. Using the definition of the diffusive flux (Eq. (1)), and Fick's law (Eq. (2)) one can show that

$$
\mathbf{v}=\mathbf{v}^{m}+D \nabla[\ln (N / \rho)]
$$

Thus the barycentric and molar velocities differ when there is a diffusive flow driven by a gradient in a total density $(N$ or $\rho)$. In the case considered in Fig. 1, the total densities are constant and both $\mathbf{v}$ and $\mathbf{v}^{m}$ vanish everywhere for purely diffusive flow. For the case of Fig. 2(a), $\mathbf{v}^{m}$ vanishes and $N$ is essentially constant. Thus Eq. 1 implies $\mathbf{J}=\rho \mathbf{v}=-D \nabla \rho$. The dashed line in Fig. 2(a) shows that this expression reproduces the observed mass flux. Note that the height dependence comes entirely from the variation in $D$ that is also evident in Fig. 11(b). If smaller values of $\epsilon_{w f} / \epsilon$ are used, the flux may actually increase at the wall. This leads to different signs on the two sides of Eq. 3!

The observed failure of the Navier condition has important consequences. Numerous arguments related to the theory of diffusive flow, such as those in Ref. [8], rely on the Navier condition to enforce a vanishing barycentric velocity in the absence of diffusion. The conclusions of these works need to be reexamined. One may wonder whether the correct boundary condition for purely diffusive flow is the identical vanishing of some other mean velocity such as $\mathbf{v}^{m}$. However, it is easy to construct exceptions to such ansatzes. The difficulty with such boundary conditions is most readily seen by considering steady state situations. Steady state implies that $\partial_{t} \rho=\partial_{t} N_{i}=0$ and the corresponding continuity equations then imply that

$$
\partial_{\alpha}\left(\rho v_{\alpha}\right)=\partial_{\alpha}\left(N v_{\alpha}^{m}\right)=0
$$


Multiplying Eq. (雨) by $N$ and taking the divergence yields

$$
\partial_{\alpha}\left(N v_{\alpha}\right)=\partial_{\alpha}\left(N v_{\alpha}^{m}\right)+\partial_{\alpha}\left(N D \partial_{\alpha}[\ln (N / \rho)]\right) .
$$

Rewriting $\partial_{\alpha}\left(N v_{\alpha}\right)$ as $\partial_{\alpha}\left[(N / \rho)\left(\rho v_{\alpha}\right)\right]$ and making use of Eq. (5), one can easily obtain

$$
v_{\alpha} \partial_{\alpha}(N / \rho)=\frac{1}{\rho} \partial_{\alpha}\left(N D \partial_{\alpha}[\ln (N / \rho)]\right) .
$$

An analogous relation for the mean molar velocity can easily be derived,

$$
v_{\alpha}^{m} \partial_{\alpha}(\rho / N)=\frac{1}{N} \partial_{\alpha}\left(\rho D \partial_{\alpha}[\ln (N / \rho)]\right) .
$$

Thus the continuum relations themselves place constraints on mean velocities. Any additional requirement that a mean velocity vanish imposes constraints on the gradients of the density. While these may be consistent with some situations, they will in general over-specify the problem.

We have tested the above equations with simulations using a range of atom sizes and masses. To illustrate their application, we use the parameters for Fig. 2(a), but adjust the probability of reflection at the osmotic wall to produce different net average mass flows down the channel. Typical examples of the resulting steady state mass density and flow profiles for $\epsilon_{w f} / \epsilon=0.3$ are shown in Fig. 2(b) and (c). Neither the barycentric nor the mean molar velocity vanishes. Indeed, they are independent of height at distances more than a molecular diameter from the wall. We find that the mean molar density $N$ is independent of $x$, and that only the $x$ components of the velocity are nonzero. Thus Eq. (8) simplifies to $v_{x}^{m}=D \partial_{x}^{2} \rho / \partial_{x} \rho$. The spatially averaged $v_{x}^{m}$ versus $\partial_{x}^{2} \rho / \partial_{x} \rho$ for several different osmotic boundary conditions is shown in Fig. 2(d). We find a good fit to a straight line whose slope $D=0.067 \pm 0.004$ agrees with the value $D=0.070 \pm 0.004$ found directly from Fick's law (Eq. (2)).

It is clear from the above examples that for multicomponent fluids the Navier condition must be modified by subtracting the diffusive flow from the left hand side of Eq.(3). We find that simulations of a variety of convective-diffusive flows can then be fit with a common value of the slip length [9].

So far we have examined only neutral wetting where both $a$ and $b$ have the same interaction with the walls. In many realistic cases, one component will preferentially wet the wall. To examine this situation we return to the case where the two particles are indistinguishable except for their labels. For particles $a(b)$ we now change the wall-fluid interaction at the top (bottom) wall so that it is purely repulsive by truncating the potential at its minimum rather than at $2.2 \sigma$. Recall that previously for this fluid we found that all average velocities were zero. Figure $3($ a) shows the average velocity produced by changing the wetting properties. One sees a remarkably strong shear flow $(\sim \mathrm{m} / \mathrm{s})$ with significant "slip" at the stationary walls. This slip has the wrong sign for the Navier condition and its magnitude is inconsistent with results for convective flows.

The driving force for the flow in Fig. 3(a) comes from the externally imposed concentration gradient and the variation of the interfacial free energy of the walls with concentration. Figure 3 (b) shows the mass fraction $c_{a}$ at different cross-sections along the channel. As can be seen from the figure, along the walls the system prefers the more strongly wetting fluid species. The difference between the concentration at the wall and in the center of the channel increases with $x$. This increase leads to a rise in interfacial tension. Values of the surface tension $\gamma$, calculated using the mechanical definition of Kirkwood and Buff [10], are shown in Fig. B(c). The boundary condition relating the shear stress on the wall $\left.\sigma_{x z}\right|_{w}$ to the viscous stress in the fluid $\left.\sigma_{x z}\right|_{f}$ is analogous to that used for Marangoni flow at a two fluid boundary [11]:

$$
\left.\sigma_{x z}\right|_{w}-\left.\sigma_{x z}\right|_{f}=\partial_{x} \gamma .
$$

It can be shown [9] that there is also a velocity discontinuity given by an integral of the Marangoni stress over the interfacial region that produces the slip in Fig. B(a). This can be incorporated into a generalized Navier slip condition by adding a source term proportional to $\partial_{x} \gamma$. These boundary conditions determine the net stress on the wall. The result is consistent with stresses measured directly in our simulations, and ranges from 50 to $75 \%$ of $\partial_{x} \gamma(\sim \mathrm{MPa})$ for the cases we have studied. This force could be used to drive a nano-motor. We have also constructed systems where both the top and bottom walls prefer the same component. In this case, one generates Poiseuille-like flow which could be used in a nano-pump.

In conclusion, we find that the flow boundary condition for convective-diffusive flow is not one of equal velocities for all species. In addition, average velocities do not, in general, vanish at the wall in the absence of viscous stress. Diffusive mass transport can contribute to a significant average velocity at the wall. Further, the presence of concentration gradients along the wall in the general case of non-neutral wetting can result in significant Marangoni-type forces which drive convective flow. These effects should be readily applicable to the design of new micro-fluidic devices and may be relevant to the function of numerous biological systems.

This material is based upon work supported by the National Science Foundation under Grant No. 0083286. We thank Intel Corporation for donating the workstations used for our simulations, which were performed using LAMMPS from Sandia National Laboratories. 
[1] P.A. Thompson and M.O. Robbins, Phys. Rev. A 41, 6830 (1990).

[2] J.-L. Barrat and L. Bocquet, Phys. Rev. Lett. 82, 4671 (1999); Faraday Discuss. 112, 119 (1999).

[3] J. Koplik and J.R. Banavar, Phys. Rev. Lett. 80, 5125 (1998).

[4] V. Ganesan and H. Brenner, Phys. Rev. Lett. 82, 1333 (1999).

[5] J. Koplik and J.R. Banavar, Phys. Rev. Lett. 82, 1334 (1999).

[6] H. Brenner and V. Ganesan, Phys. Rev. E 61, 6879 (2000).

[7] S.R. de Groot and P. Mazur, Non-Equilibrium Thermodynamics, (Dover Pub., New York, 1984).

[8] J. Camacho and H. Brenner, Ind. Eng. Chem. Res. 34, 3326 (1995).

[9] C. Denniston and M.O. Robbins, to be published.

[10] J.G. Kirkwood and F.P. Buff, J. Chem. Phys. 17, $338,(1949)$. This expression would need to be modified if our walls were not rigid.

[11] Landau and Lifshitz, Fluid Mechanics, (Pergamon Press, Oxford, 1987); S.H. Davis, Annu. Rev. Fluid Mech. 19, 403 (1987); A.A. Golovin, A.A. Nepomnyashchy and L.M. Pismen, J. Fluid Mech. 347, 317 (1997).
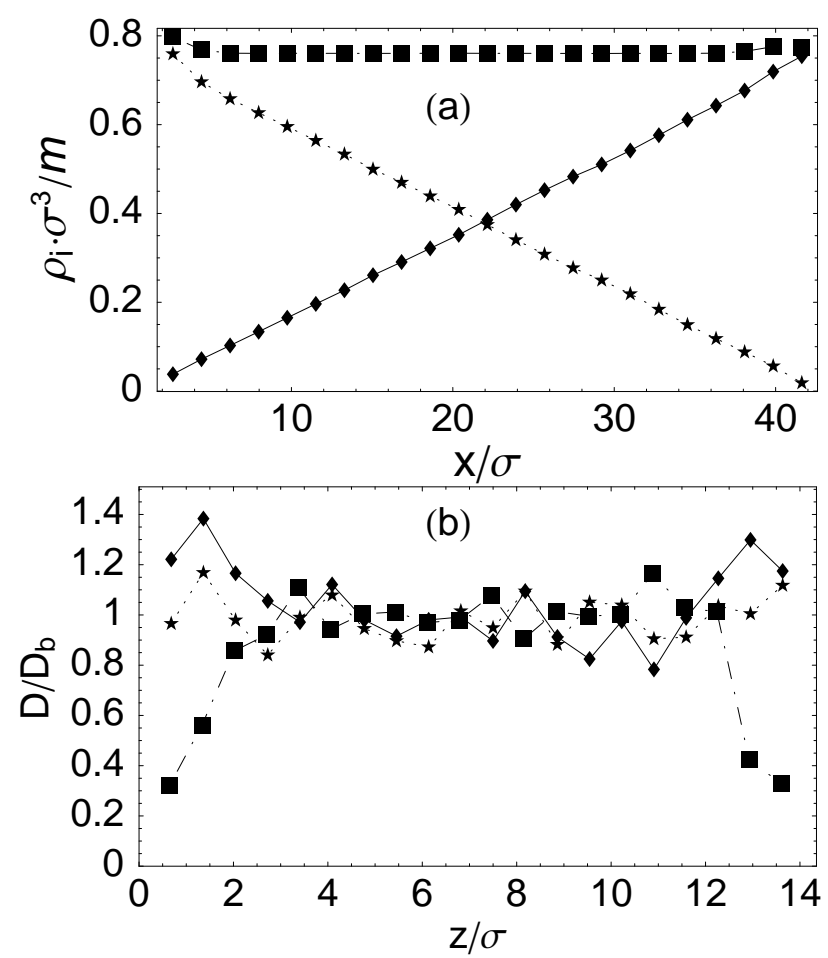

FIG. 1. (a) Density of fluid particles of type $a(\checkmark)$ and $b(\star)$, and total density ( $\mathbf{\square})$. The osmotic membrane is located at $x=0$. The periodic cell dimensions along the $x$ and $y$-directions are $42.5 \sigma$ and $12.3 \sigma$, respectively. (b) Diffusion constant for $\epsilon_{w f} / \epsilon=1.0(\boldsymbol{\square}), 0.3(\star)$, and $0.1(\diamond)$. We normalize by the bulk diffusion constant, $D_{b}=0.068 \sigma^{2} / \tau$, measured in a system without walls. Away from the osmotic membrane, the value of $D$ is independent of $x$ and $y$.
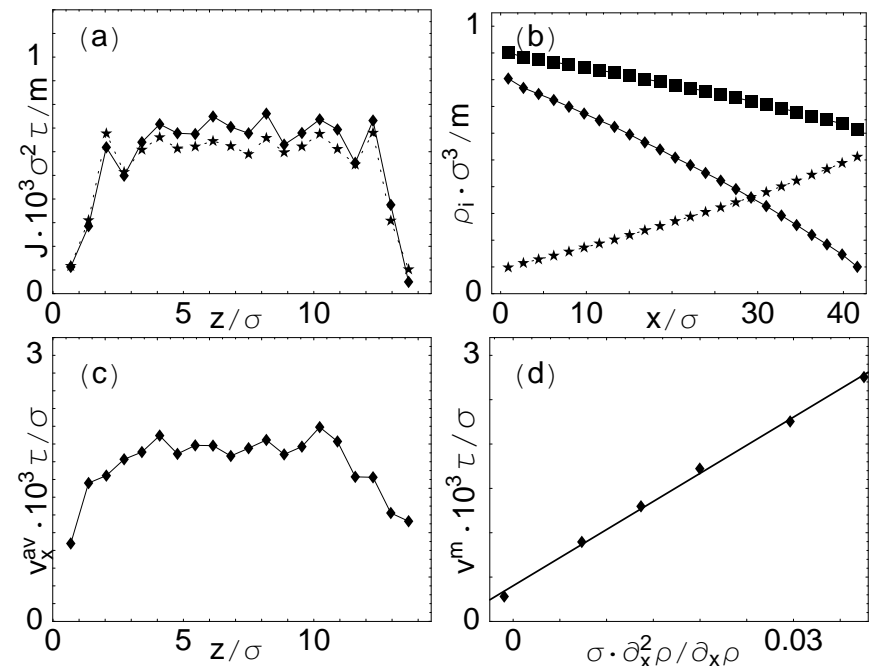

FIG. 2. (a) The observed mass flux $J=\rho v_{x}(\downarrow)$ and that predicted $J=-D \partial_{x} \rho(\star)$ by Eq. (4) for $\mathbf{v}^{m}=0$. (b) Mass density of fluid particles of type $a(\bullet)$ and $b(\star)$ and total density (ם). (c) The mass flux normalized by the mean density $\rho_{0}, v_{x}^{a v}=\rho v_{x} / \rho_{0}$. (d) Mean molar velocity versus $\partial_{x}^{2} \rho / \partial_{x} \rho$ for different osmotic walls. The line is a linear fit giving $D=0.067 \pm 0.004 \sigma^{2} / \tau$.
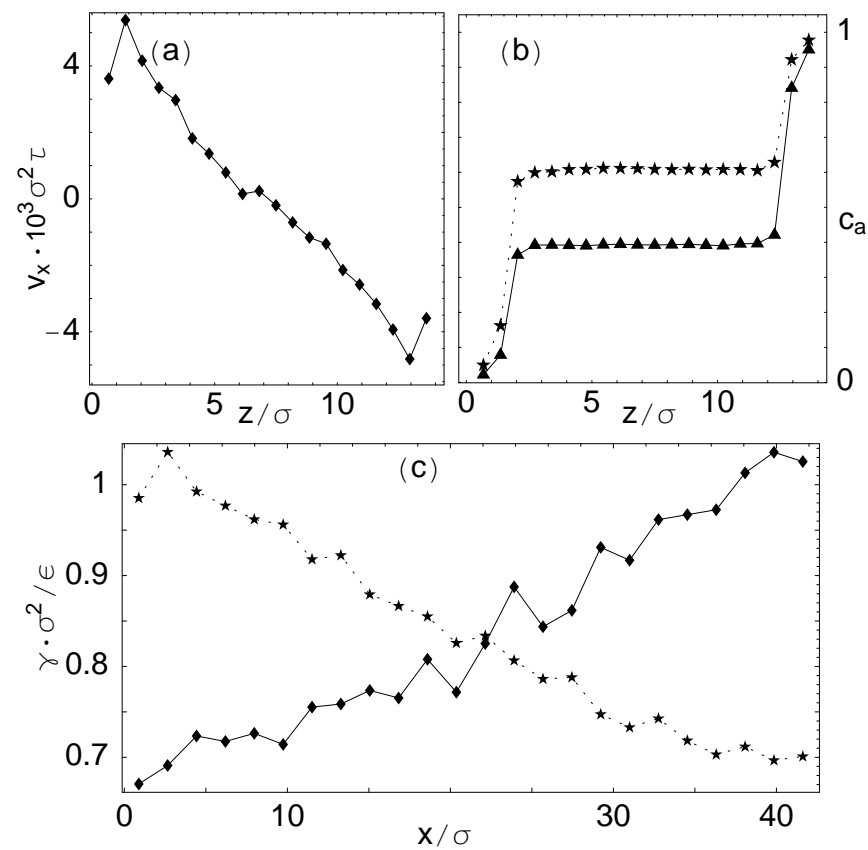

FIG. 3. (a) Barycentric velocity down the channel for a case where the wall at $z=0$ preferentially wets $a$ and the other wall preferentially wets $b$. (b) Mass fraction $c_{a}=\rho_{a} / \rho$ versus height evaluated at planes $1 / 4(\boldsymbol{\Delta})$ and $3 / 4(\star)$ of the way along the channel in the $x$-direction. (c) Surface tension along the top $(\checkmark)$ and bottom $(\star)$ walls. 